\title{
Fulvestrant in breast cancer: also a good option in triple-positive breast cancer
}

\author{
Ana López-González*,1, Irene Delgado Sillero, Mariana Lopez Flores¹, Laura \\ López-González ${ }^{1}$ \& Andres Garcia Palomo ${ }^{1}$ \\ ${ }^{1}$ Complejo Asistencial Universitario de León - Medical Oncology, C/Altos de Nava Leon, Leon 24007, Spain \\ * Author for correspondence: alopgon2@hotmail.com
}

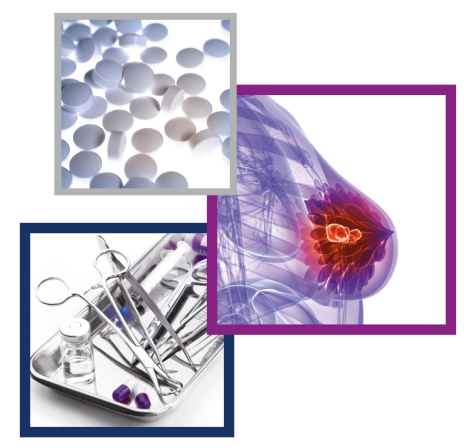

Fulvestrant is indicated in the treatment of locally advanced or metastatic breast cancer in postmenopausal women with hormone receptor-positive breast cancer as second line at the present time, after first line hormone therapy. We present here a case report of a 47-year-old woman diagnosed with infiltrating ductal carcinoma left breast cancer in 1998, stage II, luminal B HER2-positive and a liver and ganglionar relapse 3 years later. After radical treatment, fulvestrant was indicated. She has been 10 years with this treatment, with an excellent tolerance and without showing progression until now.

First draft submitted: 2 August 2017; Accepted for publication: 14 December 2017; Published online: 9 February 2018

A premenopausal 47-year-old woman was diagnosed with infiltrating ductal carcinoma left breast cancer in 1998 . The cancer was stage II, luminal B and HER2 +++ . She was treated with conservative surgery and axillar lymphadenectomy, radiotherapy and adjuvant chemotherapy with adriamycin-cyclophosphamide four cycles, and hormonal therapy with tamoxifen first and letrozole after surgical ovary ablation. She did not receive adjuvant trastuzumab because it was not the standard by the moment.

In September 2001, she had a liver metastases confirmed by punction (Figure 1). She received paclitaxeltrastuzumab. 4 months later, we found a major partial response. Radiofrequency was made and she continued with trastuzumab until November 2003. Later on, it was stopped in complete radiological response.

In May 2005, with a progression free survival of 30 months, she had a ganglionar relapse in left supraclavicular region, ER+ HER2+++. Surgical dissection was performed on the supraclavicular lymph nodes. She did not receive radiotherapy in this moment. She received trastuzumab-vinorelbin for 6 months, and then trastuzumab until a year of treatment.

The relatively short progression-free survival (PFS) could be related to absence of anti-HER2 adjuvant therapy, or indeed other prognostic factors. Chemotherapy plus anti-HER2 therapy could conceivably be considered 'adjuvant-like' therapy in the absence of objectively discernible disease.

After that, she started with fulvestrant monthly. She has been 10 years with this treatment, with an excellent tolerance and without showing progression until now.

\section{Discussion}

Fulvestrant [1] alters the dimerization of estrogen receptors, it increases their degradation, and it alters their nuclear localization.

It is indicated in the treatment of locally advanced or metastatic breast cancer in postmenopausal women with hormone receptor-positive breast cancer as second line at the present time, after first line hormonal therapy [2].

With regards to posology, the results of the CONFIRM [3,4] trial comparing fulvestrant monthly at doses of $250 \mathrm{mg}$ compared with fulvestrant dose of $500 \mathrm{mg}$ have been published and the results obtained were significant in favor of fulvestrant at doses of $500 \mathrm{mg}$, with an overall survival of 26.5 months compared with 22.3 months with a dose of $250 \mathrm{mg}$. So, fulvestrant $500 \mathrm{mg}$ intramuscular (im.) would be proposed as a standard dose fulvestrant $500 \mathrm{mg} \mathrm{im.}$

Several clinical trials have shown statistically significant results in favor of the use of fulvestrant in the first line. In the FIRST [5,6] and FALCON, Phase II and Phase III trials respectively, comparing in postmenopausal women with locally advanced or metastatic RH-positive breast cancer, as first line: fulvestrant $500 \mathrm{mg}$ im., compared with 


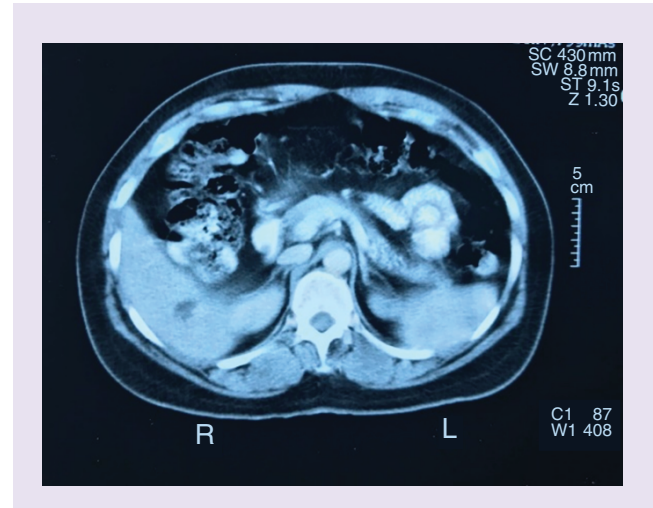

Figure 1. Liver metastasis is shown.

anastrozole $1 \mathrm{mg}$ every $24 \mathrm{~h}$; In both cases, there were statistically significant results regarding PFS in favor of fulvestrant versus anastrozole (23.5 vs 13.1 months) and overall survival (54.1 vs 48.4 months).

Other studies such as SWOG [7], which compared anastrozole monotherapy, followed by treatment with fulvestrant, versus anastrozole plus fulvestrant combined, achieved statistical significance in global survival and PFS.

The results of the clinical trial PALOMA 3 were recently reported, in which the combination of palbociclib and fulvestrant [8-10], in pre and postmenopausal women with locally advanced or metastatic breast cancer and HR-positive in progression to a previous hormone line improves PFS in comparison with fulvestrant monotherapy (9.2 vs 3.8 months).

Although fulvestrant is approved in monotherapy in progression to an anti-estrogen, there are very promising data both in the first line and in combination with palbociclib.

Our patient had received both tamoxifen and aromatase inhibitors in the adjuvant setting. After radical treatment of both relapses, treatment with fulvestrant is maintained because of the excellent tolerance and lack of progression to date.

This is a very interesting case due to a very long response to fulvestrant after two relapses. We have proposed to the patient to stop fulvestrant but she does not want to.

\section{Financial \& competing interests disclosure}

The authors have no relevant affiliations or financial involvement with any organization or entity with a financial interest in or financial conflict with the subject matter or materials discussed in the manuscript. This includes employment, consultancies, honoraria, stock ownership or options, expert testimony, grants or patents received or pending, or royalties.

No writing assistance was utilized in the production of this manuscript.

\section{Informed consent disclosure}

The authors state that they have obtained verbal and written informed consent from the patient/patients for the inclusion of their medical and treatment history within this case report.

\section{References}

1 Faslodex (fulvestrant), prescribing information. AstraZeneca Pharmaceuticals, Wilmington, DE, USA (2016).

2 NCCN Clinical Practice Guidelines in Oncology (NCCN Guidelines), Breast Cancer (version 1.2014) (2014). www.nccn.org/professionals/physician_gls/pdf/breast.pdf

3 Di Leo A, Jerusalem G, Petruzelka L et al. Final overall survival: fulvestrant $500 \mathrm{mg}$ vs $250 \mathrm{mg}$ in the randomized CONFIRM trial. J. Natl Cancer Inst. 106(1), djt337 (2014).

4 Di Leo A, Jerusalem G, Petruzelka L et al. Results of the CONFIRM Phase III trial comparing fulvestrant $250 \mathrm{mg}$ with fulvestrant $500 \mathrm{mg}$ in postmenopausal women with estrogen receptor-positive advanced breast cancer. J. Clin. Oncol. 28(30), 4594-4600 (2010).

5 Robertson JF, Llombart-Cussac A, Rolski J et al. Activity of fulvestrant $500 \mathrm{mg}$ versus anastrozole $1 \mathrm{mg}$ as first-line treatment for advanced breast cancer: results from the FIRST study. J. Clin. Oncol. 2009, 27(27), 4530-4535 (2009).

6 Ellis MJ, Llombart-Cussac A, Feltl D et al. Fulvestrant $500 \mathrm{mg}$ versus anastrozole $1 \mathrm{mg}$ for the first-line treatment of advanced breast cancer: overall survival analysis from the Phase II FIRST study. J. Clin. Oncol. 33, 3781 (2015). 
7 Mehta RS, Barlow WE, Albain KS et al. Combination anastrozole and fulvestrant in metastatic breast cancer. N. Engl. J. Med. 367, 435 (2012).

8 Turner NC, Ro J, André F et al. Palbociclib in hormone receptor-positive advanced breast cancer. N. Engl. J. Med. 373(3), 209-219 (2015).

9 Cristofanilli M, Turner NC, Bondarenko I et al. Fulvestrant plus palbociclib versus fulvestrant plus placebo for treatment of hormone-receptor-positive, HER2-negative metastatic breast cancer that progressed on previous endocrine therapy (PALOMA-3): final analysis of the multicentre, double-blind, Phase 3 randomised controlled trial. Lancet Oncol. 17, 425 (2016).

10 Harbeck N, Iyer $\mathrm{S}$, Turner $\mathrm{N}$ et al. Quality of life with palbociclib plus fulvestrant in previously treated hormone receptor-positive, HER2-negative metastatic breast cancer: patient-reported outcomes from the PALOMA-3 trial. Ann. Oncol. 27, 1047 (2016). 